\title{
Correlative TEM and FISH Imaging of Microbial Communities
}

\author{
B. Knierim*, R. Webb**, P. Wilmes***, P. Hugenholtz****, J. Liphardt*****, K. \\ McDonald******, J. Banfield*** and M. Auer* \\ * Lawrence Berkeley National Laboratory and Joint Bio-Energy Institute, 1 Cyclotron Road, \\ Mailstop 001, Berkeley, CA 94720, USA \\ ** Center for Microscopy and Microanalysis, University of Queensland, Brisbane, 4072, Australia \\ *** Dept. of Earth and Planetary Science, Hilgard Hall, UC Berkeley, Berkeley, CA 94720, USA \\ **** Joint Genome Institute, 2800 Mitchell Drive, Walnut Creek, CA 94598, USA \\ ***** Department of Physics, Stanley Hall, UC Berkeley, Berkeley, CA 94720, USA \\ ****** Electron Microscope Lab, 26 Giannini Hall, UC Berkeley, Berkeley, CA 94720, USA
}

Biofilms are the predominant lifestyle for most microbes. Mixed microbial communities are adapted to their specific environment allowing them to live under sometimes extreme conditions. They often show high species interdependencies suggesting that such communities are highly organized.

However, little is known about detailed interaction between the microbes. This is due to difficulties in both studying them at sufficient resolution to visualize the individual community members in their biofilm context and at the same time identifying each community member.

We have begun to adapt and further develop a technique that will combine TEM sample preparation and imaging with 16S rRNA Fluorescence In-Situ Hybridization (FISH) [1]. We were able to successfully label high-pressure-frozen, freeze-substituted and LR White embedded $100 \mathrm{~nm}$ biofilm sections using CARD-FISH. CARD-FISH is more sensitive compared to FISH as it involves an enhancement step, through the use of tyramide labeled-fluorophores that are locally deposited by the Horseradish Peroxidase which is bound to the primary 16S rRNA FISH probe. This resin has been shown before to be usable for CARD-FISH analysis [2] and has also provided us with the best hybridization while maintaining a good ultra structural preservation.

We are currently in the process to overlay the CARD-FISH signal onto TEM montages of $\sim 100 \mu \mathrm{m} \mathrm{x}$ $100 \mu \mathrm{m}$, recorded at $\sim 1 \mathrm{~nm}$ pixel resolution.

We applied the technique first to already well characterized Acid Mine Drainage biofilms [3], for the first time being able to determine the interactions between different microbial cells and identify them in the same samples.

The analysis of samples of the termite hindgut P3 is still in an early stage due to its much larger complexity. Our goal here is to clarify the mechanisms how the up to 200 reported species in the hindgut [4] manage to efficiently degrade lignocellulose. We further aim to complement our dual imaging approach with Raman spectromicroscopy in order to assess the cell wall decomposition of the biomass. As the termite hindgut community is regarded one of the most effective lignocellulose degrading systems, it is of great interest for the research on second generation biofuels.

We expect to gain valuable insight into the interactions of the community members as their metagenome has been established and the physiology of the community members is largely known. Moreover, we expect to see the ultrastructural inventory of extracellular machines and means of communication. 


\section{References}

[1] E. F. DeLong et al., Science 4896 (1989) 1360.

[2] O. Gros and L. C. Maurin, Acta Histochem 5 (2008) 427.

[3] P. Wilmes et al., ISME J 2 (2009) 266.

[4] F. Warnecke et al., Nature 7169 (2007) 560.

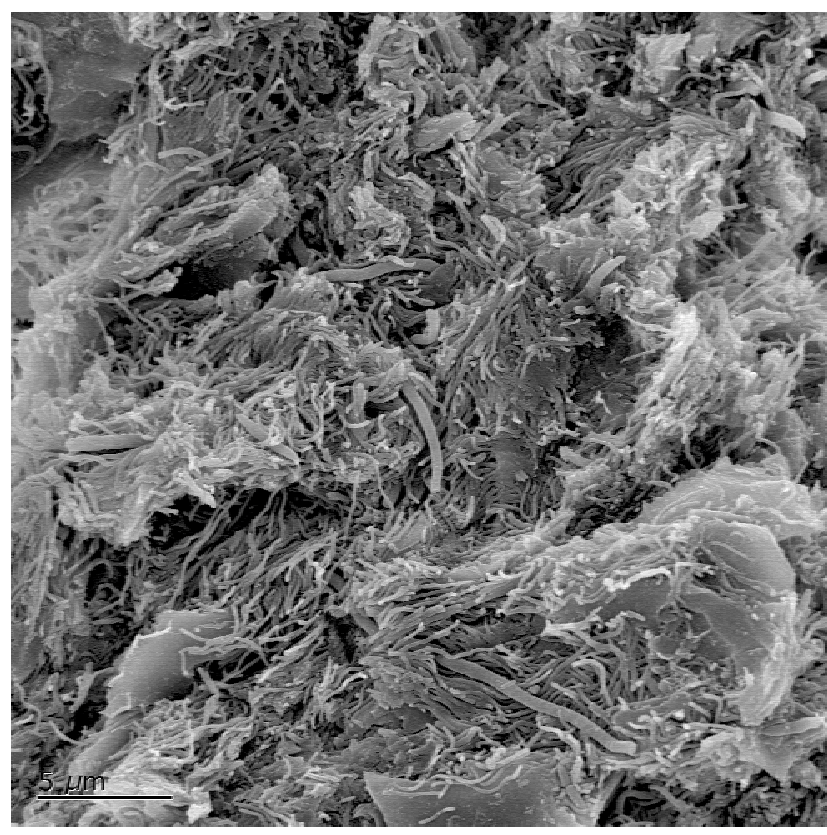

Fig. 1. SEM image of the termite hindgut microbial community, showing a huge dominance of spirochetes. Scale bar $=5 \mu \mathrm{m}$.

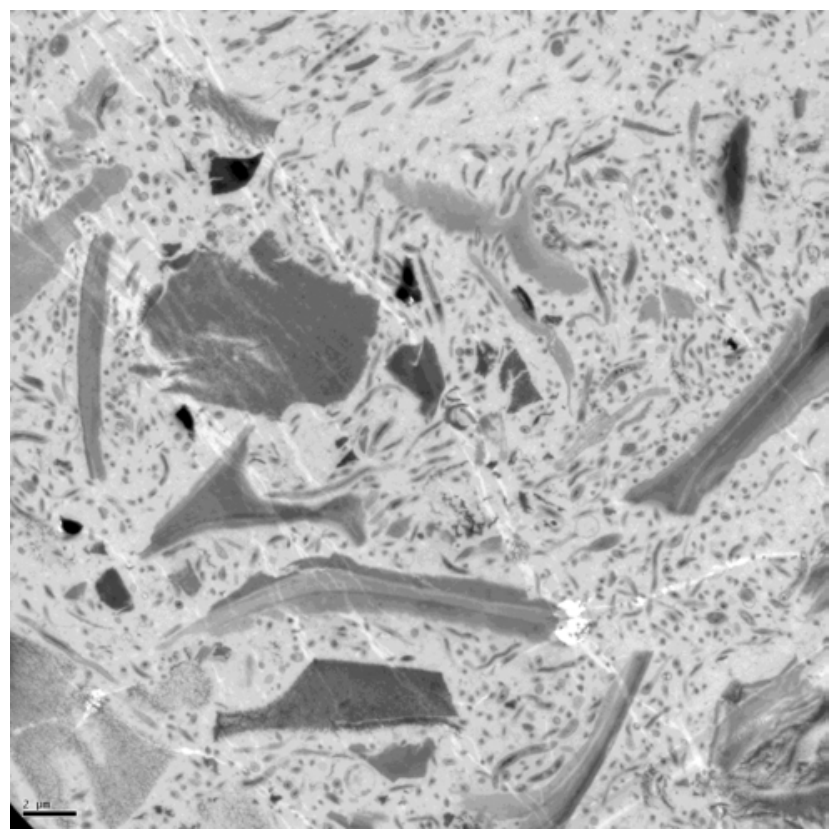

Fig. 2. Overview TEM image from a thin section of the termite hindgut microbial community. Partly digested wood particles are visible between the microbes. Scale bar $=2 \mu \mathrm{m}$ 